\title{
Deconstructing FAT
}

\section{Using memories to collectively explore implicit assumptions, values and context in practices of debiasing and discrimination-awareness}

\author{
Doris Allhutter \\ Institute of Technology Assessment \\ Austrian Academy of Sciences \\ Vienna Austria \\ dallhutt@oeaw.ac.at
}

\author{
Bettina Berendt \\ Department of Computer Science \\ TU Berlin \& KU Leuven \\ Germany \& Belgium \\ berendt@tu-berlin.de
}

\begin{abstract}
Research in fairness, accountability, and transparency (FAT) in socio-technical systems needs to take into account how practices of computing are entrenched with power relations in complex and multi-layered ways. Trying to disentangle the way in which structural discrimination and normative computational concepts and methods are intertwined, this frequently raises the question of WHO are the actors that shape technologies and research agendas - who gets to speak and to define bias, (un)fairness, and discrimination? "Deconstructing FAT" is a CRAFT workshop that aims at complicating this question by asking how "we" as researchers in FAT (often unknowingly) mobilize implicit assumptions, values and beliefs that reflect our own embeddedness in power relations, our disciplinary ways of thinking, and our historically, locally, and culturally-informed ways of solving computational problems or approaching our research. This is a vantage point to make visible and analyze the normativity of technical approaches, concepts and methods that are part of the repertoire of FAT research. Inspired by a previous international workshop [1], this CRAFT workshop engages an interdisciplinary panel of FAT researchers in a deconstruction exercise that traces the following issues:
\end{abstract}

(1) FAT research frequently speaks of social bias that is amplified by algorithmic systems, of the problem of discriminatory consequences that is to be solved, and of underprivileged or vulnerable groups that need to be protected. What does this perspectivity imply in terms of the approaches, methods and metrics that are being applied? How do methods of debiasing and discrimination-awareness enact the epistemic power of a perspective of privilege as their norm?

(2) FAT research has emphasized the need for multi- or interdisciplinary approaches to get a grip on the complex intertwining of social power relations and the normativity of

Permission to make digital or hard copies of part or all of this work for personal or classroom use is granted without fee provided that copies are not made or distributed for profit or commercial advantage and that copies bear this notice and the full citation on the first page. Copyrights for third-party components of this work must be honored. For all other uses, contact the Owner/Author.

FAT*'20, January 27-30, 2020, Barcelona, Spain

(C) 2020 Copyright is held by the owner/author(s).

ACM ISBN 978-1-4503-6936-7/20/02.

https://doi.org/10.1145/3351095.3375688 computational methods, norms and practices. Clearly, multi- and interdisciplinary research includes different normative frameworks and ways of thinking that need to be negotiated. This is complicated by the fact that these frameworks are not fully transparent and ready for reflection. What are the normative implications of interdisciplinary collaboration in FAT research?

(3) While many problems of discrimination, marginalization and exploitation can be similar across places, they can also have specific local shapes. How can FAT research e.g. consider historically grown specifics such as the effects of different colonial histories? If these specifics make patterns of discrimination have different and more nuanced dimensions than clear-cut 'redlining', what does this imply?

To explore these questions, we use the method of 'mind scripting' which is based in theories of discourse, ideology, memory and affect and aims at investigating hidden patterns of meaning making in written memories of the panelists [2]. The workshop strives to challenge some of the implicit norms and tensions in FAT research and to trigger future directions.

\section{CCS CONCEPTS}

- Applied computing - Law, social and behavioral sciences - Anthropology • Ethnography

\section{KEYWORDS}

Discrimination-awareness in machine learning, Deconstruction

\section{ACM Reference format:}

Doris Allhutter and Bettina Berendt. 2020. Deconstructing FAT: using memories to collectively explore implicit assumptions, values and context in practices of debiasing and discrimination-awareness. In Proceedings of ACM Conference on Fairness, Transparency, and Accountability (FAT* '20). ACM, Barcelona, Spain, 1 page. https://doi.org/10.1145/3351095.3375688

\section{REFERENCES}

[1] Debiasing and Discrimination-Awareness in Machine Learning: Exploring Implicit Assumptions and Context, 2018. Workshop, KU Leuven, Belgium, with D. Allhutter, B. Berendt, R. Binns, T. Calders, S. P. Gangadharan, J. Harjumaki, M. Pechenizkiy, S. Ruggieri, M. Veale and I. Žliobaitè.

[2] Doris Allhutter, 2012. Mind Scripting: A Method for Deconstructive Design. $\begin{array}{llll}\text { Science, Technology \& Human Values 37(6), 684-707. } & \text {. }\end{array}$ DOI:https://doi.org/10.1177/0162243911401633 